\title{
Formation and Obstacle Avoidance in the Unknown Environment of Multi-Robot System
}

\author{
Tao Zhang, Xiaqin Li, Yi Zhu, Song Chen, Yu Cheng and Jingyan Song \\ Department of Automation, Tsinghua University, Beijing 100084 \\ Division of Control Science and Engineering, Tsinghua National Laboratory for \\ Information Science and Technology, Beijing 100084 \\ National Key Laboratory of Flight Vehicle Control Integrated Technology, Xi' an 710065
}

China

\section{Introduction}

Nowdays, formation and obstacle avoidance issue of multi-robot system has attracted many researchers' interests due to its application background. Originally, many algorithms have been proposed for obstacle avoidance of a single robot. With the increasing requests on the application of multi-robot system for complex tasks, the obstacle avoidance of multi-robot system becomes more and more crucial (Koivo, 1998). In addition, it is found that realizing obstacle avoidance of each robot in multi-robot system is not enough. Further, it requests multiple robots to have the ability to avoid obstacle while keeping formation. If a multirobot system is in the unknown environment, it becomes more difficult to realize obstacle avoidance while keeping formation. This is just the topic of this paper.

Concerning about the formation of multi-robot system, there mainly have the following algorithms. (Ren, 2005)(Prijanian, 1999) proposed a behavior-based algorithm. In this algorithm, some expected essential behaviors are defined for robot. When the sensor of robot is stimulated by the outer environment, the output response of robot is the expected behavior according to the input of sensor information. The selection of behaviors of robot is based on some rules and each behavior has its specific purpose or task. The behavior-based algorithm can obtain real time feedback and it has complete distributed control structure. The collaboration of robot is implemented by sharing the knowledge of relative position, status, etc., among robots. Therefore, the merits of behavior-based algorithm are parallel, distributed and in real time. The disadvantage of this method is that, it is lack of clear definition of group behavior. It is hard to design the local basic behavior and control rule to synthesize nominated formation, and so on.

Another type of methods is virtual structure method (Anthony, 1997). This method assumes that there is a geometric shape among robots, which is called as virtual structure. During the movement of robots this structure is kept to fix. The merit of this method is that, it is easy to assign the group behavior of robot and it has feedback of formation. However, this method 
limits the scale of movement due to the virtual structure and the formation is therefore not so flexible.

The third type of method is leader-following algorithm (Wang, 1991). In this research, this algorithm is adopted to keep the formation due to its merits. This method will be briefly introduced in section 2 .

Concerning about obstacle avoidance, there are many algorithms. Artificial potential field algorithm is very suitable for real time control of robot due to its explicit physical implication and simple mathematical description. This method is adopted to realize obstacle avoidance in this research. The fundamental idea will be introduced in section 3 .

This paper presents formation and obstacle avoidance in the unknown environment of multi-robot system. With the leader-following algorithm, multi-robot system can form and keep various formations and realize formation transformation. Through artificial potential field algorithm, multi-robot system can successfully avoid obstacle in the unknown environment while keeping formation. The proposed method not only can realize obstacle avoidance of multi-robot system with formation in the unknown environment, but also can be adopted in real time and applicable to many different situations. By means of Pioneer3 mobile robot platform, the simulation and experimental works, which considering various formations and unknown environments, demonstrate the effectiveness of the proposed method.

The organization of the chapter is as below. In section 2, the multi-robot system formation by means of leader-following algorithm is introduced. Section 3 presents the obstacle avoidance of multi-robot system with formation in the unknown environment by means of artificial potential field method. Section 4 shows the simulation and experimental results by use of Pioneer 3 mobile robot platform. In the last section $\mathrm{V}$ the conclusion is drawn.

\section{Multi-robot formation by means of leader-following algorithm}

\subsection{Leader-following algorithm}

Leader-following algorithm is first proposed in (Wang, 1991), which is adopted for the formation control of mobile robot. The fundamental idea is that, in a group composed of multiple robots, one of robots is assigned as the leader and others are followers. The followers are keeping the position and direction with a certain distance to the leader. According to the relative position between leader and follower, it can form different network topology structures, i.e., different formations. In this algorithm, the collaboration of multi-robots is implemented based on the knowledge about the status of leader robot. The leader can control the motion trend of the system. Each follower is controlled with a formation through keeping the limitation of distance, direction angle, etc., with the leader.

The leader-following algorithm can be applicable to various situations. For example, either one leader or more leaders can be assigned. But the leader of the formation of group must be only one. Since the leader-following algorithm is based on the space geometric relation among robots, the merit of this algorithm is that, the behavior of the entire robot group can be controlled as long as the behavior or trajectory of the leader is given. It can simplify the control process of multi-robot system. However, this algorithm has two obvious disadvantages. One is that, there is no clear formation feedback because the leader does not consider other robots' movement during the course of motion. For example, if the leader moves too fast, or the follower is blocked by obstacle, the formation will be destroyed. Under the serious condition, the quality of fulfilling the task may be affected. The second 
disadvantage is that, if the leader is failed, the entire formation can not be kept and the consequence will be very serious.

The leader-following algorithm is mainly adopted for the flight of spacecrafts with formation, the collaboration of robot manipulators in the production line, as well as searching in a region by multiple robots, etc.

\subsection{Behavior of leader for formation}

The task of leader is to lead the entire formation to the destination. By means of the idea of behavior-based algorithm, the control of leader has three behaviors. One is destinationoriented forward walking behavior. The second is obstacle avoidance behavior, which will be introduced in the next section. The third is lock-prevent behavior. The highest priority of the above three behaviors is lock-prevent behavior. And the lowest priority of them is destination-oriented forward walking behavior.

\section{Destination-oriented forward walking behavior}

In the destination-oriented forward walking behavior, a robot can be regarded as particle. The destination position of leader is defined as $\left(x_{f g}, y_{f g}\right)$. The current position is $\left(x_{c}, y_{c}\right)$. When leader does not reach the destination position, that is $\left(x_{f g}-x_{c}\right)^{2}+\left(y_{f g}-y_{c}\right)^{2}>\varepsilon$, the velocity of the leader is as below.

$$
V_{L}=\frac{V}{\sqrt{\left(x_{f g}-x_{c}\right)^{2}+\left(y_{f g}-y_{c}\right)^{2}}}\left[\begin{array}{l}
x_{f g}-x_{c} \\
y_{f g}-y_{c}
\end{array}\right]
$$

\section{Lock-prevent behavior}

In the process of robot movement to the destination in the environment with obstacles, robot may not continuously move and can not fulfil the task. At this moment, robot is staying at the status of locking. In order to assist robot escape from this status, it is better to define a lock-prevent behavior.

In this research, Pioneer3 mobile robot is adopted to test the proposed method. This kind of robot has defined a lock-prevent behavior, called ArActionStallRecover. When the wheel of the robot is locked, it can move by means of a series of pre-defined actions. Normally, this behavior is set with the highest priority.

\subsection{Behavior of follower for formation}

The task of follower is to reach the destination following the leader. It only needs to follow the leader and be able to avoid the obstacle, without knowing the position of destination and the route of formation. Therefore, one of important behaviors of follower is to keep the formation. According to (Shao, 2005), the motion function of single mobile robot is as below.

$$
\left[\begin{array}{l}
\dot{x}(t) \\
\dot{y}(t) \\
\dot{\phi}(t)
\end{array}\right]=\left[\begin{array}{cc}
\cos \phi(t) & 0 \\
\sin \phi(t) & 0 \\
0 & 1
\end{array}\right]\left[\begin{array}{l}
v(t) \\
\omega(t)
\end{array}\right]
$$


where $(x, y, \phi)$ denotes the robot position and motion direction in a certain inertial coordinate system. $(v, \omega)$ denotes the linear velocity and angular velocity of moving object. A certain point $h$ at the place which has the distance $L$ from the axis line of robot to the center of the object is considered as below. The reason to consider this point is that, this point is always the central position of the sensor of moving object in the experimental platform. The offset point of this axis center is defined as,

$$
\left\{\begin{array}{c}
x_{h}=x+L \sin \phi \\
y_{h}=y+L \cos \phi \\
\phi_{h}=\phi
\end{array}\right.
$$

It can be obtained as below after equation transformation.

$$
\left[\begin{array}{l}
\dot{x}_{h}(t) \\
\dot{y}_{h}(t) \\
\dot{\phi}_{h}(t)
\end{array}\right]=\left[\begin{array}{cc}
\cos \phi(t) & -L \sin \phi(t) \\
\sin \phi(t) & L \cos \phi t) \\
0 & 1
\end{array}\right]\left[\begin{array}{l}
v(t) \\
\omega(t)
\end{array}\right]
$$

Based on the above model, a simple leader-following system, illustrated by Fig.1, can be considered, where $R_{L}$ denotes leader, $R_{F}$ follower.

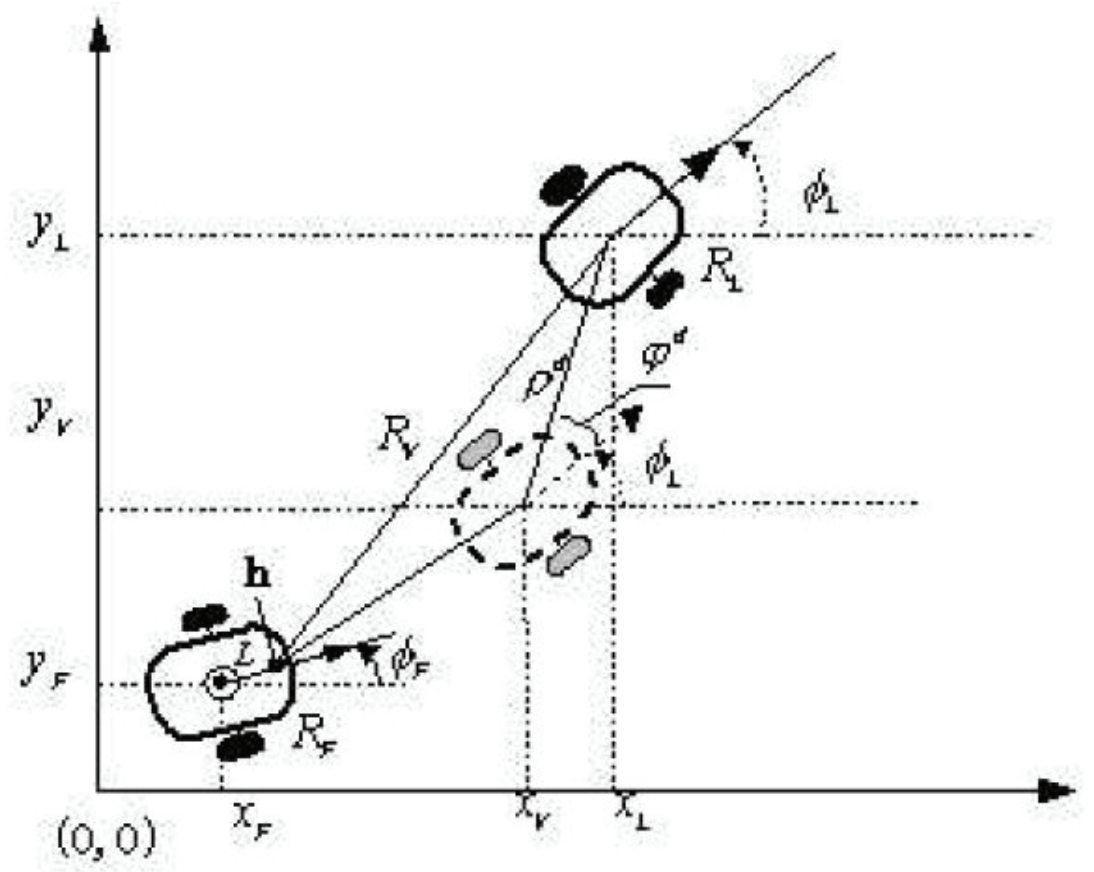

Fig. 1. Simple model of leader-following system 
Since the aim of control is to keep the pre-defined distance $\rho^{d}$ and angle $\varphi^{d}$ between the follower $R_{F}$ and the leader $R_{L}$, it can be assumed that there is a virtual leader $R_{V}$ in the expected formation of the follower $R_{F}$. Therefore, what we need to do is to control the follower $R_{F}$ to move following the virtual leader $R_{V}$. By referring to leader $R_{L}$, the motion function of $R_{V}$ is as below.

$$
\begin{aligned}
& \dot{x}_{V}=v_{L} \cos \phi_{L}+\rho^{d} \sin \left(\varphi^{d}+\phi_{L}\right) \phi_{L} \\
& \dot{y}_{V}=v_{L} \sin \phi_{L}-\rho^{d} \cos \left(\varphi^{d}+\phi_{L}\right) \phi_{L} \\
& \phi_{V}=\omega_{L}
\end{aligned}
$$

Therefore, the tracking error between $R_{F}$ and $R_{V}$ is

$$
\begin{aligned}
& \dot{\bar{x}}=v_{L} \cos \phi_{L}-v_{F} \cos \phi_{F}+\rho^{d} \sin \left(\phi^{d}+\phi_{L}\right) \dot{\phi}_{L}+L \dot{\phi}_{F} \sin \phi_{F} \\
& \dot{\bar{y}}=v_{L} \sin \phi_{L}-v_{F} \sin \phi_{F}-\rho^{d} \cos \left(\phi^{d}+\phi_{L}\right) \dot{\phi}_{L}-L \dot{\phi}_{L} \cos \phi_{F} \\
& \dot{\bar{\phi}}=\omega_{L}-\omega_{F}
\end{aligned}
$$

Where $\bar{x}=x_{V}-x_{F}, \bar{y}=y_{V}-y_{F}, \bar{\phi}=\phi_{V}-\phi_{F}$

The next step is to choose a control law to reduce the tracking error. The control law is selected as below.

$$
\begin{aligned}
& v_{F}=v_{L} \cos \bar{\phi}+k_{1}\left(\rho \cos \varphi-\rho^{d} \cos \left(\varphi^{d}+\bar{\phi}\right)\right)+\rho^{d} \omega_{L} \sin \left(\varphi^{d}+\bar{\phi}\right) \\
& \omega_{F}=\frac{1}{L}\left[v_{L} \sin \bar{\phi}-k_{2}\left(\rho \sin \varphi-\rho^{d} \sin \left(\varphi^{d}+\bar{\phi}\right)\right)-\rho^{d} \omega_{L} \cos \left(\varphi^{d}+\bar{\phi}\right)\right]
\end{aligned}
$$

(Shao, 2005) has proved the convergence of this algorithm.

\section{Obstacle avoidance of multi-robot system with formation}

\subsection{Behavior of leader for obstacle avoidance}

The behavior of leader for obstacle avoidance is based on artificial potential field (APF) algorithm. The APF algorithm is proposed by Khatib in 1986 (Khatib, 1986). Originally, this method is to solve the problem that manipulator can not bumps into working table when it moves to grasp the object. Later it is found that this method has good effect for mobile robot. It can generate very smooth moving trajectory. The basic idea of this method is to simplify the robot, obstacle and target to be a point respectively. Therefore, the moving space of robot becomes a two-dimensional space. If robot wants to reach the destination, it needs to continuously move to the destination. This movement process can be regarded as a motion in a virtual artificial field of force. The obstacle can generate repulsion to robot and the target point can generate attraction. The resultant of attraction and repulsion can control the moving direction of robot. Any moving direction of any position of robot in the space is determined by the total strengthen of field composed by repulsion field of obstacle and attraction field of target point. The attraction position field function is defined as,

$$
\mathrm{U}_{\mathrm{att}}(\mathrm{X})=\mathrm{k} \rho^{2}\left(\mathrm{X}, \mathrm{X}_{\mathrm{g}}\right)
$$


$k$ is positive ratio gain coefficient of position. $X, X_{g}$ respectively denote the position of robot and target point at the moving space. $\rho\left(X, X_{g}\right)=\left\|X-X_{g}\right\|$ denotes the $F_{\text {att }}$ distance between robot and target point. The attraction force of target point is the negative gradient of attraction potential field. It is as below.

$$
\mathrm{F}_{\mathrm{att}}=-\nabla\left[\mathrm{U}_{\mathrm{att}}(\mathrm{X})\right]=\mathrm{k} \rho\left(\mathrm{X}, \mathrm{X}_{\mathrm{g}}\right)
$$

The repulsion potential field function is as,

$$
\mathrm{U}_{\mathrm{reg}}(\mathrm{X})=\left\{\begin{array}{cc}
\eta\left(\frac{1}{\rho\left(\mathrm{X}, \mathrm{X}_{0}\right)}-\frac{1}{\rho_{0}}\right)^{2} & \rho\left(X, \mathrm{X}_{0}\right) \leq \rho_{0} \\
0 & \rho\left(X, X_{0}\right) \geq \rho_{0}
\end{array}\right.
$$

$\eta$ is positive ratio gain coefficient of position. $\rho\left(X, X_{0}\right)$ is the shortest distance between robot and obstacle in the moving space. $\rho_{0}$ is the biggest distance of obstacle affecting the robot. Its value is determined based on the specific situation between obstacle and target point. Normally, this value is smaller than the half of the distance among each obstacle and the smallest distance between target point and each obstacle. When robot does not reach the target point, the repulsion of obstacle to the target point is as,

$$
\mathrm{F}_{\text {rep }}(\mathrm{X})=-\nabla\left[\mathrm{U}_{\text {rep }}(\mathrm{X})\right]\left\{\begin{array}{cc}
-\frac{2 \eta}{\rho(X, X)^{2}}\left(\frac{1}{\rho\left(\mathrm{X}, \mathrm{X}_{0}\right)}-\frac{1}{\rho_{0}}\right) & \rho\left(X, X_{0}\right) \leq \rho_{0} \\
0 & \rho\left(X, X_{0}\right) \geq \rho_{0}
\end{array}\right.
$$

The resultant force of robot is as,

$$
F=F_{\text {att }}+F_{\text {rep }}
$$

This resultant force determines the moving direction. The graph of force is illustrated as below.

\subsection{Behavior of follower for obstacle avoidance}

Concerning about obstacle avoidance, assume that each robot has one virtual outer covering. The radius of this outer covering is determined by the maximal velocity of robot as well as braking ability. It is therefore called as protected shell. Any object and other robots should be avoided to enter into the region of this protected shell.

If follower detects out one obstacle in this protected shell, illustrated by Fig.3, it will turn a small angle to avoid the direction of this obstacle. And then it will turn an angle which is necessary to keep the formation. These two angles are combined to calculate the new expected angle $\varphi^{\prime d}$. The calculation equation is as below.

$$
\varphi^{\prime d}=\varphi^{d}-\frac{\varphi^{d}-\gamma}{\left\|\varphi^{d}-\gamma\right\|} \arccos \sqrt{1-\frac{\left(l^{d}-l\right)^{2}}{2\left(\rho^{d}\right)^{2}}}
$$

where $l$ denotes the shortest distance between the center of follower and obstacle. $\gamma$ denotes the relative angle of the nearest point between the center of robot and obstacle. According to this strategy, follower can not be collided with the nearest obstacle while keeping the expected formation. 


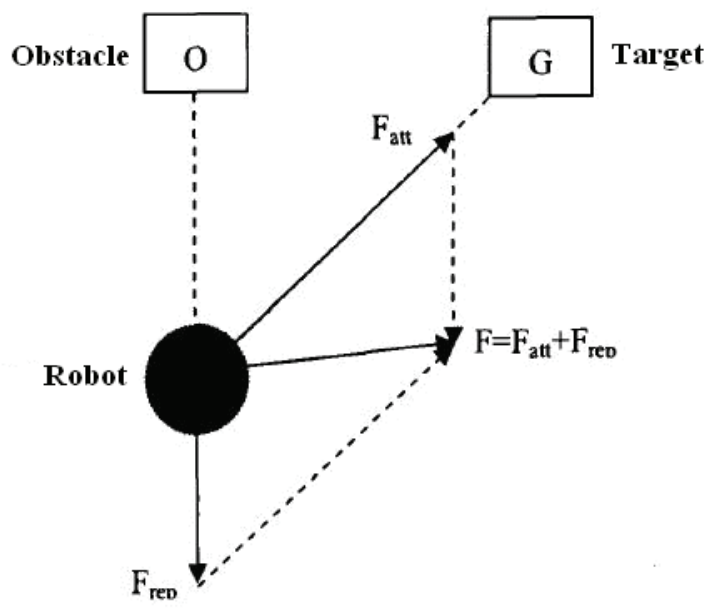

Fig. 2. Force of robot in the artificial potential field

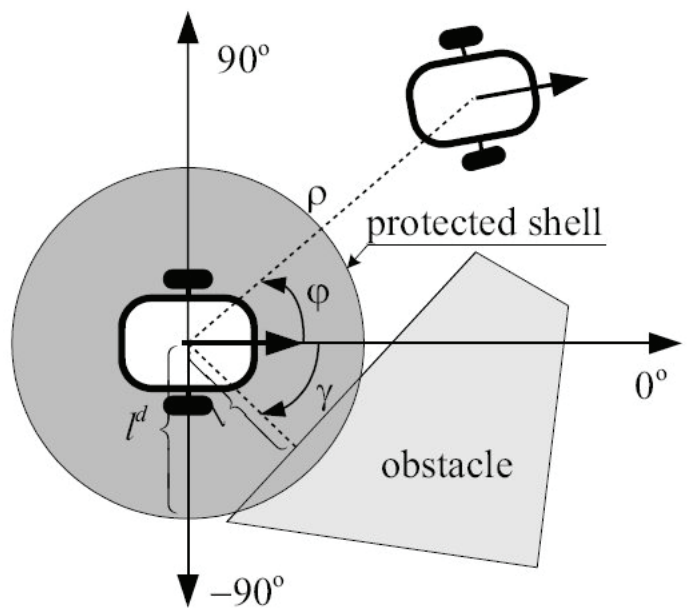

Fig. 3. Obstacle avoidance of follower

\section{Simulation and experimental results}

\subsection{Pioneer3 mobile robot platform}

As the simulation and experimental platform, Pioneer3 mobile robot is adopted, which has sonar and hodometers. Sonar is used as the device of measuring distance from obstacle to robot. Hodometer is used as the positioning device to obtain the current position relating to the starting point in real time. Pioneer3 robot has totally 2 sonar cycles, respectively at the front part and rear part of robot. Each sonar cycle has 8 transducers. In this research 8 transducers of front part of sonar cycle and 2 transducers of rear part of sonar cycle are used. The following Fig.4 is the sonar distribution and its identity coordinate of Pioneer3. When positioning concretely, the identity coordinate of Pioneer3 at the starting moment is 
regarded as the global coordinate for this path planning. In each simulation figure, $G$ denotes target and S starting point.

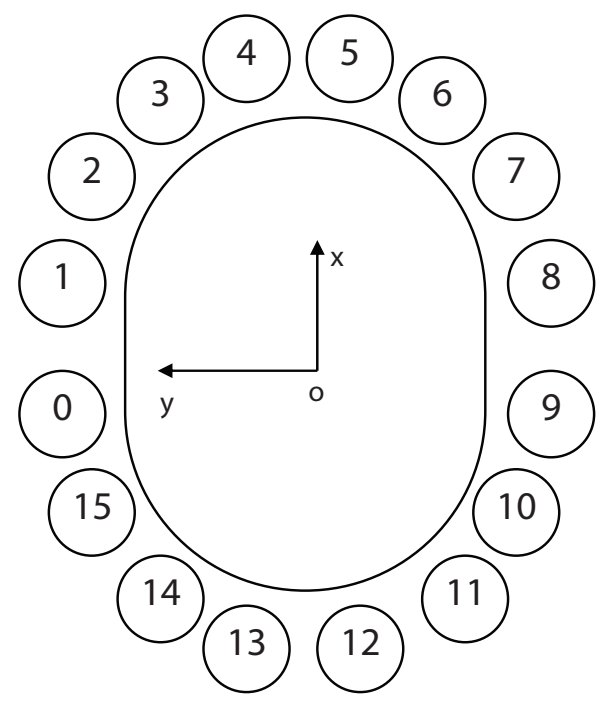

Fig. 4. Sonar distribution and its identity coordinate of Pioneer3

\subsection{Simulation and experimental results}

Pioneer3 mobile robot platform provides a simulation system MobileSim. In this system, a map can be installed and sensor information can be obtained. User can run the developed program in MobileSim before running on Pioneer3 mobile robot. Actually, it has same effect of running in MobileSim and Pioneer3 mobile robot.

In order to verify the proposed method, the following simulation and experimental works have been carried out by use of MobileSim.

Fig.5 is the simulation results about the obstacle avoidance of leader robot individually.

Fig.6 Illustrates the formation transformation among various formations in the environment without obstacle.

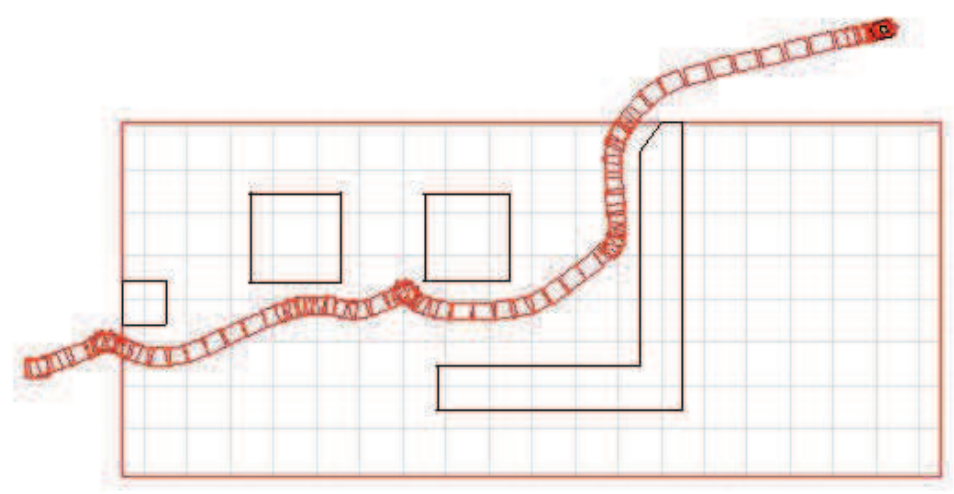

Fig.5 Obstacle avoidance of leader robot individually 


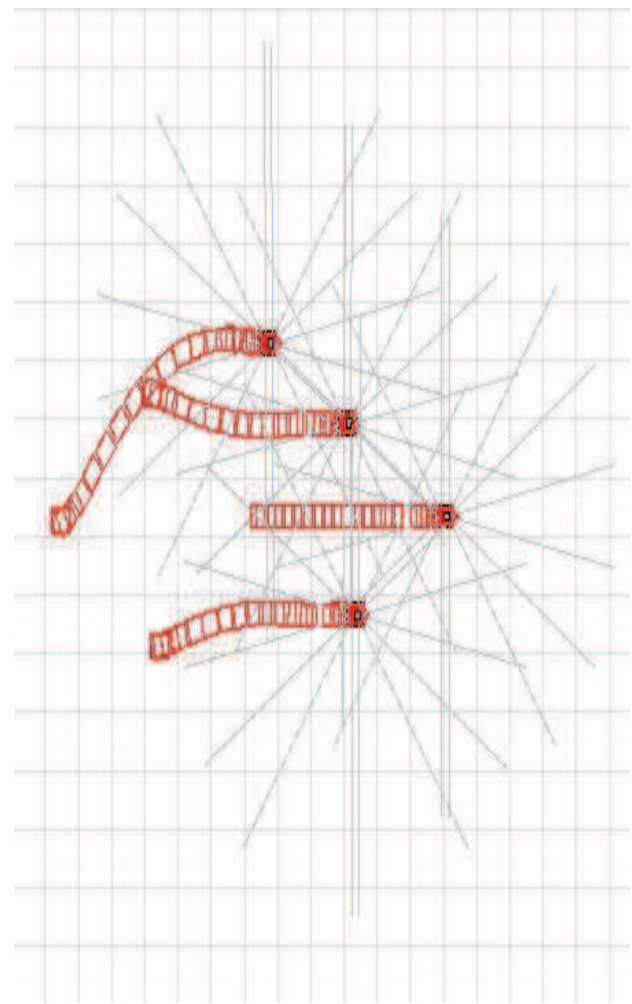

(a) From diamond to triangular formation

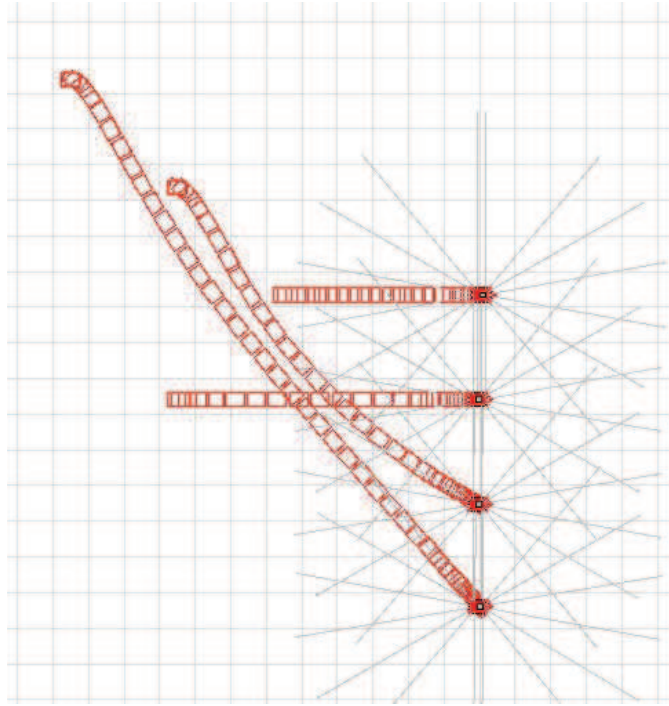

(b) From triangular to straight line formation 


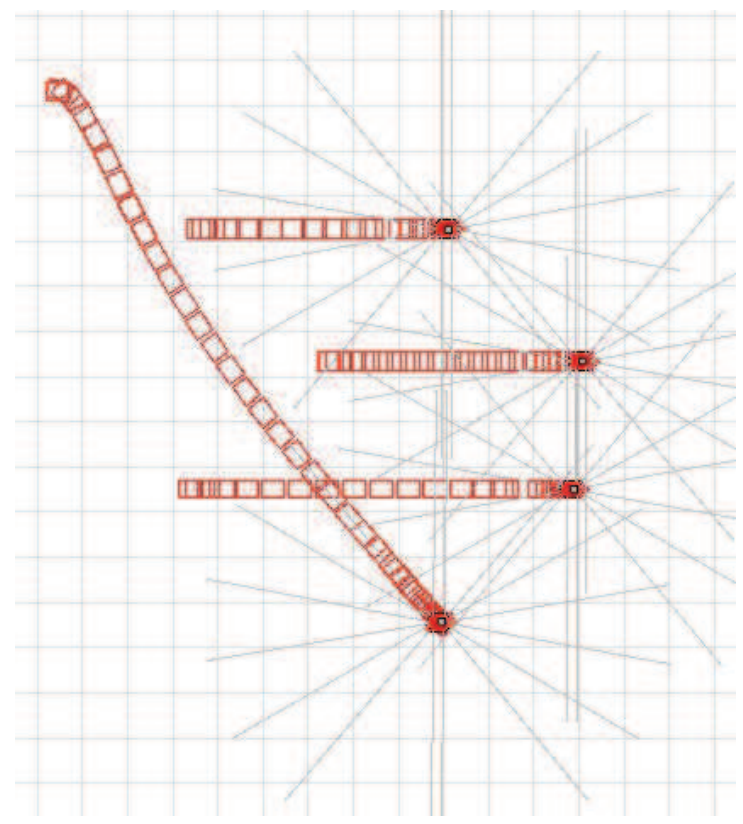

(c) From triangular to trapezoid formation

Fig. 6. Formation transformation

Fig.7 illustrates the simulation results of obstacle avoidance of two robots with formation.

Fig.8 illustrates the experimental results of the proposed method in the Pioneer3 mobile robot.

\section{Conclusions}

This paper introduces formation and obstacle avoidance in the unknown environment of multi-robot system. With the leader-following algorithm, multi-robot system can form and keep various formations and realize formation transformation. Through artificial potential field algorithm, multi-robot system can successfully avoid obstacle in the unknown environment while keeping formation. The proposed method not only can realize obstacle avoidance of multi-robot system with formation in the unknown environment, but also can be adopted in real time and applicable to many different situations. By means of Pioneer3 mobile robot platform, the simulation and experimental works, which considering various formations and unknown environment, demonstrate the effectiveness of the proposed method. In the future research, we will further consider more complicated environment with various types of obstacles. In addition, we will propose new method to optimize the movement of multiple robots with formation in order to improve autonomous and adaptive features of multi-robot system.

\section{References}

Anthony, L. M. \& Tan, K. H. (1997). High precision formation control of mobile robots using virtual structures autonomous, Autonomuous Robots, Vol. 4, pp.260-266. 


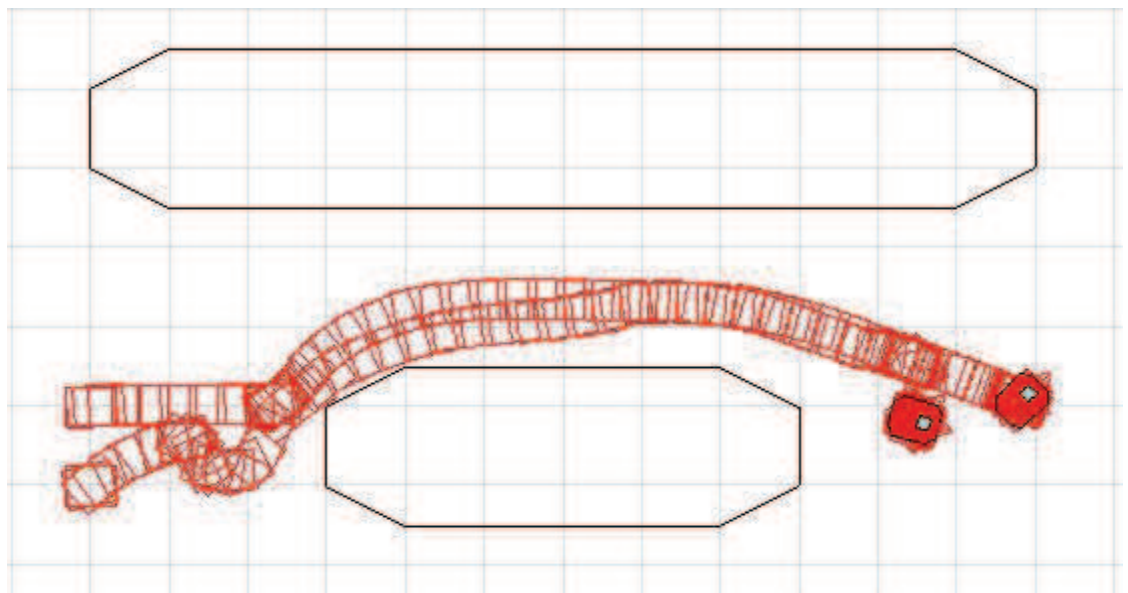

(a) Passing through a channel of two robots

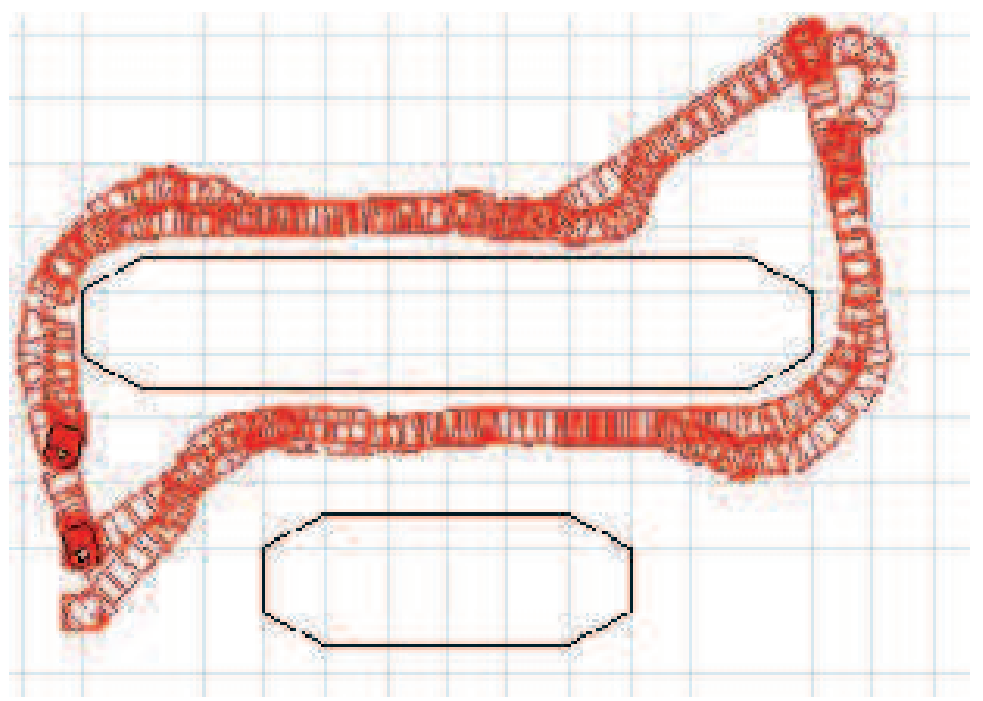

(b) Going there and back of two robots

Fig. 7. Obstacle avoidance of two robots

Khatib, O. (1986). Real-time obstacle avoidance for manipulators and mobile robot, International Journal of Robotics Research, Vol. 5, No. 1, pp. 90-98.

Koivo, A. J. \& Bekey, G. A. (1998). Report of workshop on coordinated multiple robots: planning, control and application, Robotic and Automation, Vol. 14, No. 1, pp.91-93.

Pirjanian. (1999). Behavior coordination mechanisms state of the art, Technical Report, Institute of Robotics and Intelligent Systems, School of Engineering, University of Southern California.

Ren, D. H. \& Lu, G. Z. (2005). Consideration on formation control, Control and Decision Making, Vol. 20, No. 6, pp.601-606. (in Chinese) 


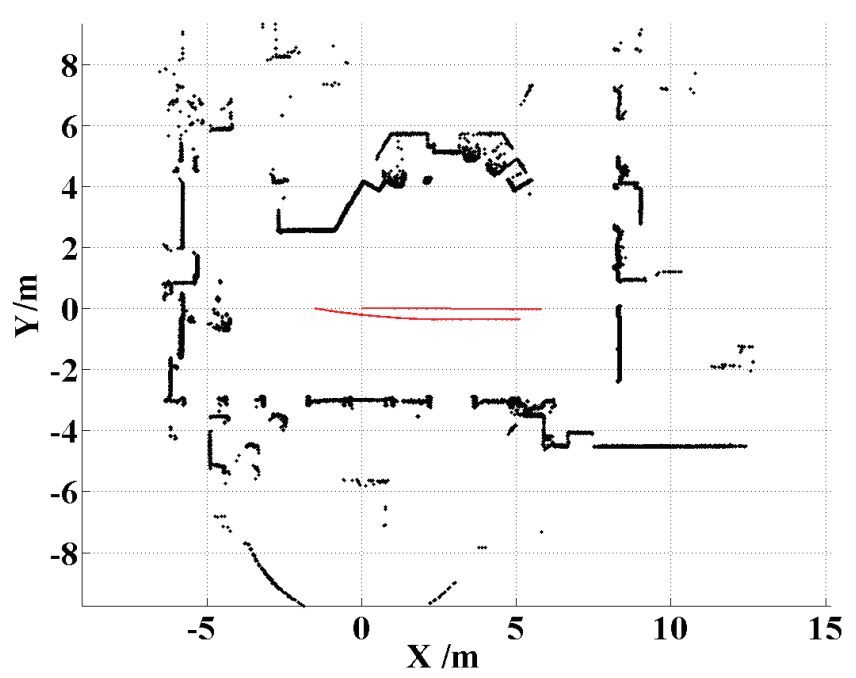

(a) Formation of two robots

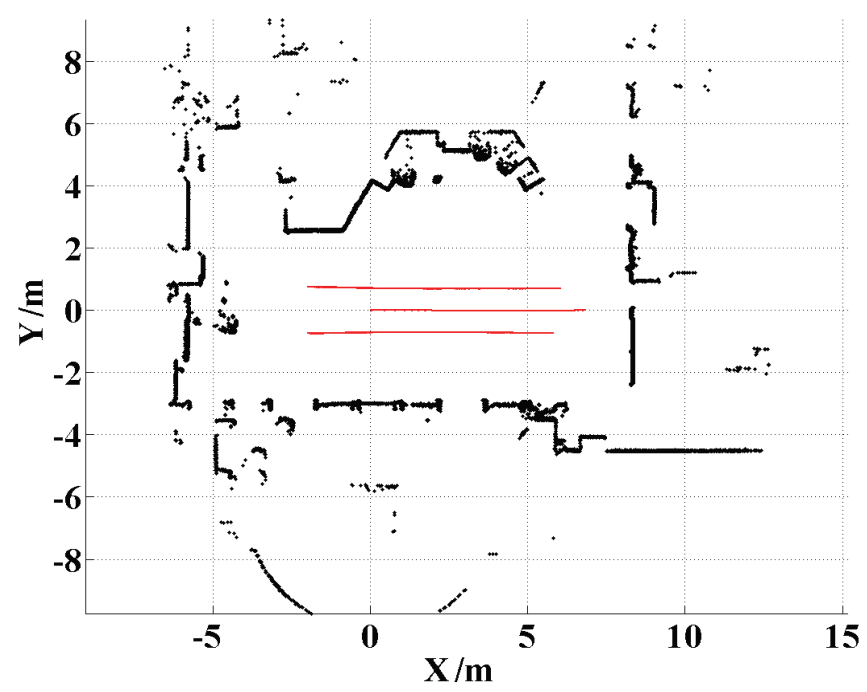

(b) Formation of three robots

Fig. 8. Experimental results

Shao, J.; Xie, G.; Yu, J.; et al. (2005). Leader-following formation control of multiple mobile robots, Proceedings of the 2005 IEEE International Symposium on Intelligent Control, pp.808-814.

Wang, P. K. C. (1991). Navigation strategies for multiple autonomous mobile robots moving in formation, Journal of Robotic Systems, Vol. 8, No. 2, pp. 177-195. 


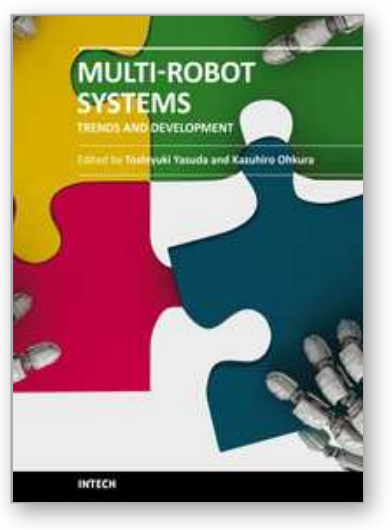

\author{
Multi-Robot Systems, Trends and Development \\ Edited by Dr Toshiyuki Yasuda
}

ISBN 978-953-307-425-2

Hard cover, 586 pages

Publisher InTech

Published online 30, January, 2011

Published in print edition January, 2011

This book is a collection of 29 excellent works and comprised of three sections: task oriented approach, bio inspired approach, and modeling/design. In the first section, applications on formation, localization/mapping, and planning are introduced. The second section is on behavior-based approach by means of artificial intelligence techniques. The last section includes research articles on development of architectures and control systems.

\title{
How to reference
}

In order to correctly reference this scholarly work, feel free to copy and paste the following:

Tao Zhang, Xiaqin Li, Yi Zhu, Song Chen, Yu Cheng and Jingyan Song (2011). Formation and Obstacle Avoidance in the Unknown Environment of Multi-Robot System, Multi-Robot Systems, Trends and Development, Dr Toshiyuki Yasuda (Ed.), ISBN: 978-953-307-425-2, InTech, Available from: http://www.intechopen.com/books/multi-robot-systems-trends-and-development/formation-and-obstacleavoidance-in-the-unknown-environment-of-multi-robot-system

\section{INTECH}

open science | open minds

\section{InTech Europe}

University Campus STeP Ri

Slavka Krautzeka 83/A

51000 Rijeka, Croatia

Phone: +385 (51) 770447

Fax: +385 (51) 686166

www.intechopen.com

\section{InTech China}

Unit 405, Office Block, Hotel Equatorial Shanghai

No.65, Yan An Road (West), Shanghai, 200040, China

中国上海市延安西路65号上海国际贵都大饭店办公楼 405 单元

Phone: +86-21-62489820

Fax: +86-21-62489821 
(C) 2011 The Author(s). Licensee IntechOpen. This chapter is distributed under the terms of the Creative Commons Attribution-NonCommercialShareAlike-3.0 License, which permits use, distribution and reproduction for non-commercial purposes, provided the original is properly cited and derivative works building on this content are distributed under the same license. 